\title{
Mobility Extension for Broadband Satellite Multimedia
}

\author{
Y. Fun $\mathrm{Hu}$, Prashant Pillai \\ School of Engineering, Design \& Technology \\ University of Bradford \\ Bradford, West Yorkshire, United Kingdom \\ \{y.f.hu, p.pillai\}@bradford.ac.uk
}

\author{
Matteo Beriolli \\ Institute of Communications and Navigation \\ German Aerospace Center (DLR) \\ Oberpfaffenhofen-Wessling, Germany \\ matteo.berioli@dlr.de
}

\begin{abstract}
This paper presents an extension for Broadband Satellite Multimedia (BSM) to support Mobility Management (MM). To support seamless handovers, mobility related entities are proposed in the Satellite Independent Access Function (SIAF) defined over the SI-SAP interface. This enables the mapping of the mobility related primitives between the higher layer and the satellite dependant lower layers via the Satellite Dependent Access Function (SDAF). The proposed MM architecture incorporates the Media Independent Handover Services defined in the IEEE802.21 to support handover between different satellite systems. This includes definition of the Media Independent Handover Function (MIHF) which provides interworking between heterogeneous 802 networks and between 802 and other terrestrial mobile networks through the support of three services for handover, namely, Media Independent Event Services (MIES), Media Independent Command Services (MICS) and Media Independent Information Services (MIIS). A detailed description of the function architecture and a case study showing the signalling interaction between MIH and SI-SAP primitves are presented.
\end{abstract}

Keywords-BSM, mobility, handover management, MIH

\section{INTRODUCTION}

The Broadband Satellite Multimedia (BSM) architecture [1] standardised by ETSI defines satellite independent services and related functionalities to provide efficient IP-based broadband services over satellite. The BSM architecture defines the Satellite Independent Service Access Point (SI-SAP) interface that separates the satellite independent features of the upper layers from the satellite dependant features of the lower layers, and provides a mechanism to carry IP based protocols over these satellite-dependent lower layers. This enables not only the integration of satellite networks with fixed and mobile network infrastructures, but also allows interoperability at the IP layer between satellite systems of different physical and link layers technologies with full compliance to the SI-SAP concept.

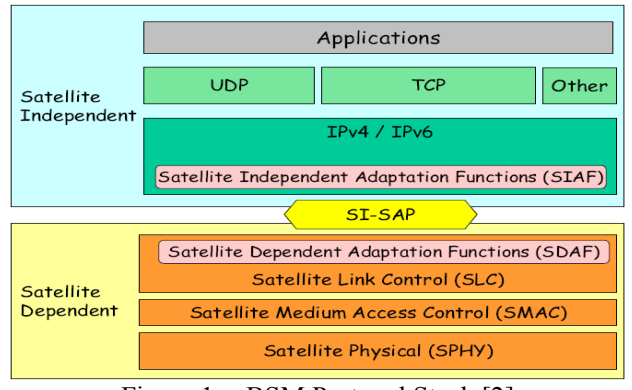

Figure 1. BSM Protocol Stack [2]
There has been an increasing user need for broadband services on the move. However, currently the BSM architecture does not support mobility functions. To support such broadband mobile services through satellites, a mobility extension for BSM is required. The need for mobility was described and a generic mobility extension for BSM was proposed in [3]. This paper further expands on this work and provides a more detailed description of the proposed MM architecture that incorporates the IEEE802.21 MIH framework. Following this section, Section II gives an overview of the IEEE802.21 MIH framework and its associated services. Section III describes an MIH-enabled mobility management architecture for BSM. Section III describes various BSM handover management scenarios and possible handover strategies. Section V provides a list of MIH-enabled mobility primitives for BSM. A case study of handover between a BSM network and a 802.16 network is presented in Section VI. This is followed by a conclusion in Section VII.

\section{MEDIA INDEPENDENT HANDOVER FRAMEWORK}

The IEEE802.21 Media Independent Handover (MIH) framework defines a unified interface between different link layer technologies for the support of seamless mobility between heterogeneous IEEE 802 networks and between IEEE 802 and other mobile wireless technologies. This unified interface is presented as an abstraction layer function, the Media Independent Handover Function (MIHF), for handover detection, initiation and decision via Layer 2 triggers. Figure 2 shows the IEEE802.21 MIHF reference model and SAPs [4].

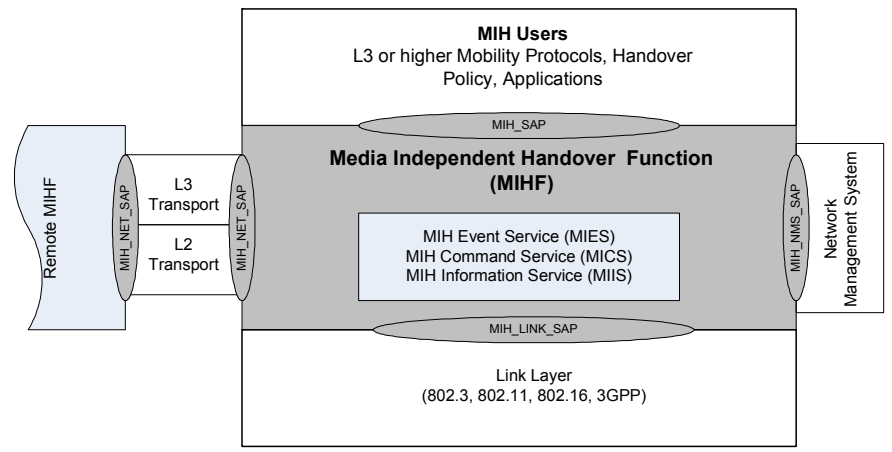

Figure 2. General IEEE802.21 MIHF Reference Model and SAPs [5]

An MIHF in a network entity that communicates directly with an MIHF in a mobile node acts as a Point of Service (PoS) of that mobile node (MN). The MN exchanges $\mathrm{MIH}$ information with its MIH PoS using L3 transport if the PoS 
does not reside in the same network entity as its network Point of Attachment (PoA). A PoA is the network side of a layer 2 link that includes an $\mathrm{MN}$ as the other end point. Thus, the MIH framework supports both L2 and L3 transports for exchanging MIH information.

To facilitate media independent handover, the MIHF provides three services: Media Independent Event Service (MIES), Media Independent Command Services (MICS) and Media Independent Information Services (MIIS). The MIES reports events on dynamic changes in link characteristics, links status and link quality to upper layers through the MIHF. The MICS is used to gather information about the status of the connected links. Upon reception of event notification, $\mathrm{MIH}$ users make use of the MICS to pass link commands to the lower layers via the MIHF to manage and control the link layer behaviour for handover decision. MIIS provides the capability for obtaining the necessary information for handovers, including neighbouring networks, link layer information and service availability. This information will be used to assist network discovery and selection to enable more effective handover. Entities that use the services provided by the MIHF are called MIH users. MIH users access MIHF services through a variety of SAPs. Each SAP consists of a set of service primitives that specify the interactions between the service user and provider. Three SAPs are currently defined within the MIH framework: the MIH_SAP for the Upper Layer access to the Lower Layers via the MIHF; the MIH LINK SAP to connect the MIHF and the Lower Layers, and MIH_NET_SAP for service transport between the local and the remote MIHFs.

- MIH_SAP: A media independent interface provides the interface between the MIHF and the upper layers of the mobility management protocol stack. In order to receive MIHF generated events and link layer events that are forwarded by the MIHF, the upper layers need to subscribe with the MIHF as MIH users. MIH users can directly send commands to the local MIHF using the service primitives of the MIH_SAP.

- MIH_LINK_SAP: An abstract media dependent interface between the MIHF and media specific lower layer to allow MIHF to use services from the lower layers of the mobility management protocol stack. For each link layer technology, the MIH_LINK_SAP maps to the media specific SAPs.

- MIH_NET_SAP: An abstract media dependent interface of the $\bar{M} I H F$, provides transport services over the data plane on the local node to support the exchange of $\mathrm{MIH}$ information and messages with remote MIHFs. Transport services provided by the MIH_NET_SAP can use either L2 or L3 signalling.

The MIH_NMS_SAP as shown in Figure 2 was originally proposed to be included in the MIH generic reference model [5] and a set of primitives for MIH_NMS_SAP has been defined in [6]. However since MIHF makes use of existing management SAPs defined for specific link layer technologies, the MIH_NMS_SAP was not included in the current version of the $802 . \overline{2} 1$ standard. For example, the MLME_SAP defines the interface between the MIHF and the management plane of an IEEE 802.11 network, whereas the M_SAP which is defined in
IEEE 802.16 provides the interface between the MIHF and the IEEE 802.16 management plane functions.

\section{MIH-BASED MOBILITY ARCHITECTECTURE FOR BSM}

The BSM SI-SAP and the IEEE802.21 MIHF both consider the separation of technology-independent upper layers from the technology-dependent lower layers to enable interoperability among heterogeneous networks. Figure 3 shows the proposed functional architecture for BSM mobility management, incorporating the IEEE 802.21 MIH framework. In this framework the primitives defined in the SAPs of the MIH framework are adopted for SI-SAP mobility support. In addition, the term MN used in the MIH framework will be replaced by the term BSM terminal hereafter.

MIH users can make use of the Event Subscription mechanism as defined in the MIH framework to selectively receive either Link Events or MIH Events or both for handover events notifications. A new functional entity, the Mobility Handler, is created in the SIAF. The Mobility Handler will perform higher layer mobility functions and will translate IP mobility functions into BSM mobility functions and vice versa. It is logically connected to the MIH_SAP and acts as the MIHF to provide MIHF services to MIH users via the MIH_SAP. A BSM Mobility Management functional block can be included, if required, to handle BSM specific mobility management support in the lower layer. The mobility handler is connected to the BSM Mobility Management entity in the SDAF through the SI-SAP to access BSM-specific mobility services supported by the SDAF. Such a mobility architecture enables handover between different BSM networks through the SI-SAP as well as between BSM networks and other non-BSM networks through interaction between the MIH_SAP and the SI-SAP.

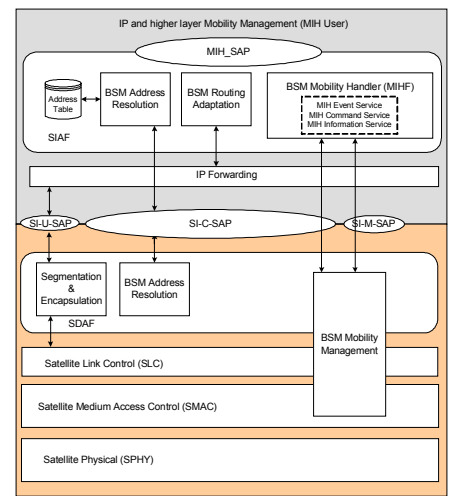

Figure 3. Functional Architecture for BSM Mobility Management

The mobility handler interacts with the BSM QoS management and BSM QoS adaptation functional blocks to performed resource allocation functions and with the security management functional block to perform authentication and access control functions during the handover execution process when connections/sessions need to be established with the target network. In relation to location management, the BSM Address Resolution block is used for mapping BSM addresses to IP addresses when a handover from the serving BSM PoS to the target BSM PoS belonging to a different subnet is required. The BSM Routing Adaptation block is used for facilitating mobility related routing in order to make sure incoming and outgoing packets to and from the BSM terminal can be routed 
to the new PoS where the BSM terminal is attached. The Routing Adaptation also facilitates a smooth handover where packets are forwarded between the serving PoS and the target PoS.

\section{BSM HANDOVER MANAGEMENT AND STRATEGY}

BSM handover can be categorised into intra-BSM network handover, inter-BSM networks handover and handover between BSM and non-BSM networks. Intra-BSM network handover refers to handover within a BSM network (e.g. within a DVB-RCS network); whereas inter-BSM network handover refers to handover between two different BSM networks. Both types of handover can involve beam handover, satellite handover and gateway handover. For intra-BSM network handover, the handover procedures will be handled inside the BSM network with specific SD signalling and procedures, and it is outside the scope of a general BSM MM, and thus will be not considered in this paper. For inter-BSM handover, the SISAP layer will provide adaptation between the different link layer technologies. If the handover involves a gateway handover, services provided by the MIHF can be utilised as this involves higher layer mobility management functions. Similarly for handover between BSM and non-BSM networks, both SI-SAP and MIHF services will be exploited. This paper concentrates on handover between BSM and non-BSM networks.

In general, there are three handover phases: the handover initiation phase, the handover decision phase and handover execution phase. Very often, a mobile terminal moves between satellite coverage areas causing a handover. Normally such movement is anticipated through the geographical position change and/or variation of the link quality between the mobile terminal and the network. Other circumstances may include service access from another serving area. In the handover initiation phase, relevant parameters will be collected to monitor the status of the systems in real time. These parameters will be processed and examined so that a handover decision can be made. The handover decision phase is responsible for QoS negotiation if necessary, for example, the candidate network cannot provide the same QoS supported by the serving network. The handover execution phase is responsible for the actual handover implementation including path creating and switching, route optimization, and handover completion.

To perform handover, different strategies can be implemented, namely, Mobile Control Handover (MCHO), Mobile Assisted Handover (MAHO), Network Control Handover (NCHO) and Network Assisted Handover (NAHO). In the MCHO scheme, the mobile terminal is responsible for monitoring and collecting the downlink information and subsequently makes handover decision based on such information. This scheme facilitates a faster, though less reliable, handover decision process with no additional signalling from the network to the BSM terminal but at the expense of a much higher terminal complexity. In the MAHO scheme, both the mobile terminal and the network make measurements and monitor the radio link parameters from the uplink and downlink. In this case, the terminal will pass the monitored and measured information from the current and target downlink to the network periodically for further analysis and for making handover decision. The interval of sending downlink measurement information to the network from the terminal has to be selected properly. If the interval is too short, an excessive signalling load will be generated, while with too long an interval, the handover response will be too slow. An appropriate interval should be chosen in order to permit a rapid response when a handover is required. In the $\mathrm{NCHO}$, the network carries sole responsibility for monitoring and collecting the required information and data from the current radio links and surrounding radio links which can be the candidates for handover implementation and the mobile terminal is not involved in the handover initiation and decision phases. The network will make a handover decision based on those measurements. With this scheme, the network periodically measures the link condition in the uplink and when the signal level falls below a certain handover threshold the network initiates a handover process. It is also possible that the network will initiate a handover based on network load condition or location information rather than the radio link information. In the NAHO scheme, the mobile terminal and network will monitor and collect downlink and uplink information respectively. Handover decision is made by the mobile terminal based on its own downlink measurement as well as the uplink measurements delivered from the network. Thus, handover reliability is increased. However, the signalling load is higher than the $\mathrm{NCHO}$ and MCHO since the required uplink information has to be sent to the mobile terminal from the network.

\section{MIH-ENABLED BSM HANDOVER PRIMITIVES}

Table I shows a set of SI-SAP service primitives for mobility support in both the control and management planes that map onto the MIH_Link SAP. SI-SAP primitives defined in the management plane correspond to the service management primitives defined in the MIH framework and the control plane primitives to the MIH event service primitives.

\begin{tabular}{|l|l|}
\hline MIH_LINK_SAP Primitives & $\begin{array}{l}\text { Corresponding SI-SAP } \\
\text { Primitives }\end{array}$ \\
\hline Link_Detected.indication & SI_C_LinkDetected.ind \\
\hline Link_Up.indication & SI_C_LinkUp.ind \\
\hline Link_down.indication & SI_C_LinkDown.ind \\
\hline Link_Parameters_Report.indication & SI_C_MEFReport.ind \\
\hline Link_Going_Down.indication & SI_C_LinkGoingDown.ind \\
\hline Link_Handover_Imminent.indication & SI_C_HO.ind \\
\hline Link_Handover_Complete.indication & SI_C_HC.ind \\
\hline Link_PDU_Transmit_Status.indication & SI_C_PDUTxStatus.ind \\
\hline Link_Actions.request & SI_C_HO.req \\
Link_Actions.confirm & SI_C_HO.cfm \\
\hline Link_Get_Parameters.request & SI_C_GetMEF.req \\
Link_Get_Parameters.confirm & SI_C_GetMEF.cfm \\
\hline Link_Capability_Discover.request & SI_M_CapDiscover.req \\
Link_Capability_Discover.confirm & SI_M_CapDiscover.cfm \\
\hline Link_Event_Subscribe.request & SI_M_EvntSub.req \\
Link_Event_Subscribe.confirm & SI_M_EvntSub.cfm \\
\hline Link_Event_Unsubscribe.request & SI_M_EvntUnsub.req \\
Link_Event_Unsubscribe.confirm & SI_M_EvntUnsub.cfm \\
\hline Link_Configure_Thresholds.request & SI_M_ConfigThd.req \\
Link_Configure_Thresholds.confirm & SI_M_ConfigThd.cfm \\
\hline
\end{tabular}

Table I SI-SAP mobility service primitives

Currently, SI-SAP service primitives in relation to QoS, Address and Routing as well as mulitcast have been defined. These SI-SAP primitives will be reused whenever QoS 
parameters and routing and switching functions are considered or required during the handover initiation/decision/execution processes. It is also envisaged that more management primitives will be defined in future. However, these will not be addressed in this paper. Although most parameters contained in these SI-SAP mobility primitives will be similar to those in the corresponding MIH_Link_SAP primitives, mapping of parameters between these two SAPs may be required.

\section{CASE STUdy: HANDOVER BETWEEN BSM AND 802.16 NETWORKS}

In order to demonstrate how MIH can be incorporated in the SI-SAP protocol stack, Figures $4 \mathrm{a}$ and $4 \mathrm{~b}$ show the signaling flow diagrams for the handover initiation and execution phases from a BSM (DVB-RCS) network to a 802.16 network using NAHO. The signaling flows are adapted from [4] and may not be optimized for signaling efficiency. In addition, only signaling relevant to inter-operations between $\mathrm{MIH}$ primitives and SI-SAP primitives are shown. It has to be noted that the MIHF/SIAF functional block in the signaling diagrams represents the BSM mobility handler as shown in Figure 3. The procedure is summarized as follows:

\section{Handover Initiation}

The signaling flows for the handover initiation phase includes procedure for Measurement Report, Information Query, Link Going Down Indication and Scan for Candidate Network as shown in Figure 4a. The MIH user in the BSM terminal receives link measurement report from the MIHF through the MIH LINK Parameters Report.Indication primitives via the SI_C_MEFReport.ind from the SDAF. Neighbouring networks information is then acquired through the MIH_GET_Information.Request/Confirm message. Upon notification of a link going down event through the SI_C_LinkDown.ind primitive from the SDAF, the mobility handēer generates the MIH_LINK_Going_Down.Indication primitive, which is then passed to the $\bar{M} I H$ User. Upon receiving the link down event, a MIH_LINK_Actions is requested from the MIH user to the mobility handler, which in turn informs the SDAF of an imminent handover decision. A series of events is then triggered to scan the link status of the candidate networks (candidate networks 1 and $n$ respectively in the signaling diagram). Relevant parameters are acquired from the candidate network.

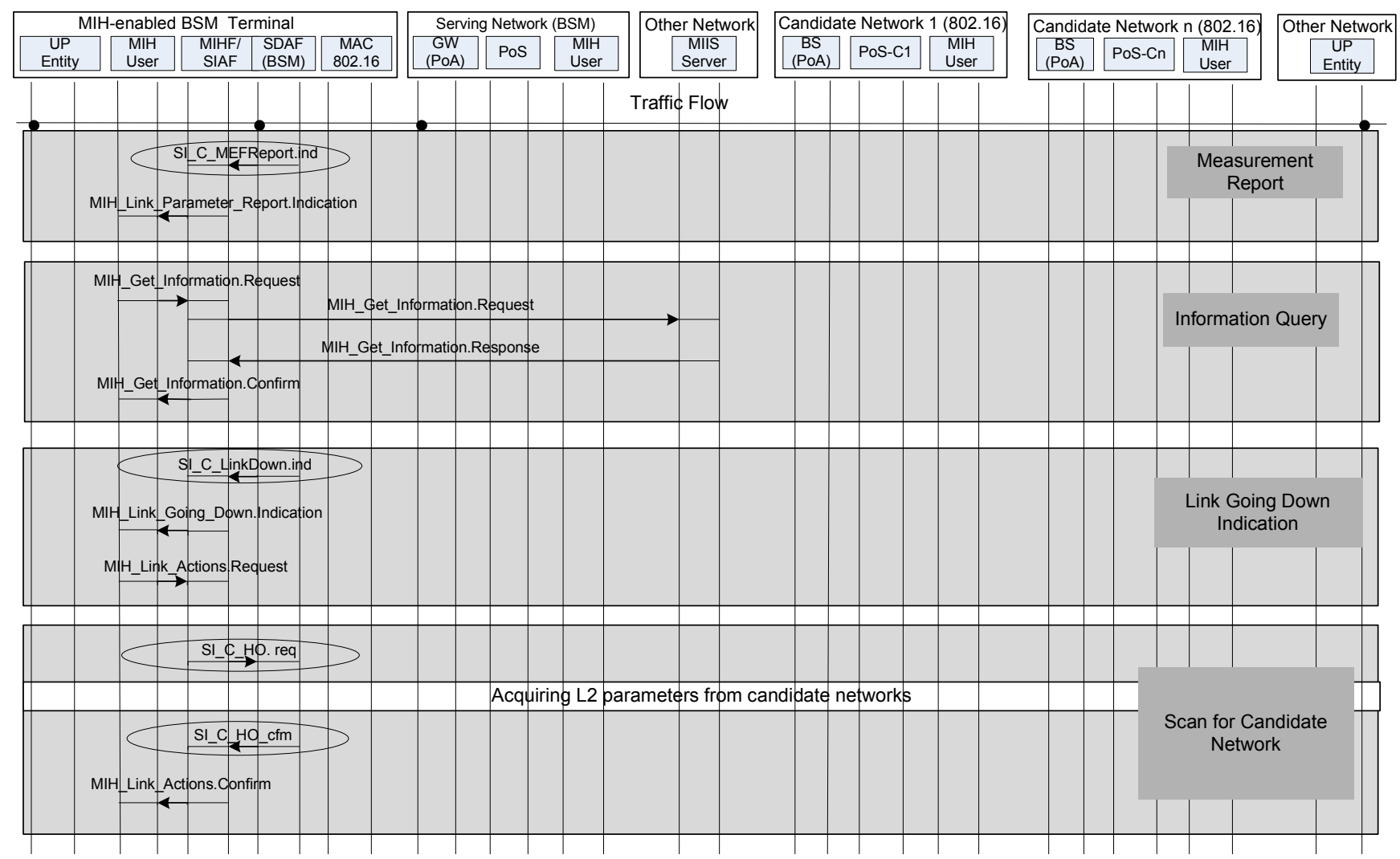

Figure 4a Handover Initiation for handover from MIH-enabled BSM network to 802.16 network

\section{Handover Decision and Preparation}

In this phase, the BSM terminal identifies the resource availability status of the candidate network by sending the MIH_MN_HO_Candidate_Query message to the serving PoS.
When the Serving PoS receives the MIH_MN_HO_Candidate Query.Request from the BSM terminal, it retrieves resource information from target network by sending MIH_N2N_HO_Query_Resources message to the PoSs on the candidate networks and then based on resource availability and 
other selection criteria, a target network is identified. The BSM terminal then sends the MIH_MN_HO_Commit.Request message to the serving PoS to notify it of the target network information. The serving $\mathrm{PoS}$ reserves the resources the resource at the target through $\mathrm{MIH}$ N2N_HO_Commit messages. Once the resources are reserved, the $\overline{\mathrm{MI}} \mathrm{HF}$ sends the MIH_MN_HO.Confirm primitive to the MIH user for the next handover phase. No SI-SAP primitives/messages are involved. Readers can refer to [4] for signalling in relation to resource availability check and preparation for this handover phase.

\section{Handover Execution and Completion}

Upon receiving the message $\mathrm{MIH}$ MN_HO.Confirm primitive, the MIH user sends a MIH_MN_HO.Request to the MIHF, which is translated into the SI $\bar{C}$ HO.req primitive through the SIAF and then passed to the SDAF to trigger a series of message exchanges with the target 802.16 network to establish a new layer 2 connection. Once the new link is established, the SI_C_HO primitive will be passed from the SDAF to SIAF, from which the MIH_LINK Actions.Confirm primitive will be passed to the MIH user. Higher layer mobility procedure (mobile IP) is then carried out and the session is now switched over to the 802.16 network. The BSM terminal then sends the MIH_MN_HO_Complete.Request message to the serving PoS on the 802.16 network and the serving PoS exchanges the MIH_N2N_HO_Complete messages with the previous PoS on the BSM network to release the resource that was reserved for the BSM terminal.

\section{CONCLUSION}

In this paper, a BSM mobility architecture incorporated with the MIH framework to enable handover between BSM networks and non-BSM networks has been derived. SI-SAP primitives compatible with the MIH_LINK_SAP primitives for mobility support have been defined. Different BSM handover scenarios and possible handover strategies have been described. Finally, a signalling chart for handover of an active session from a MIH-enabled BSM network to a 802.16 network is presented in order to demonstrate how these two standard interfaces can be utilised for handover between satellite and terrestrial networks.

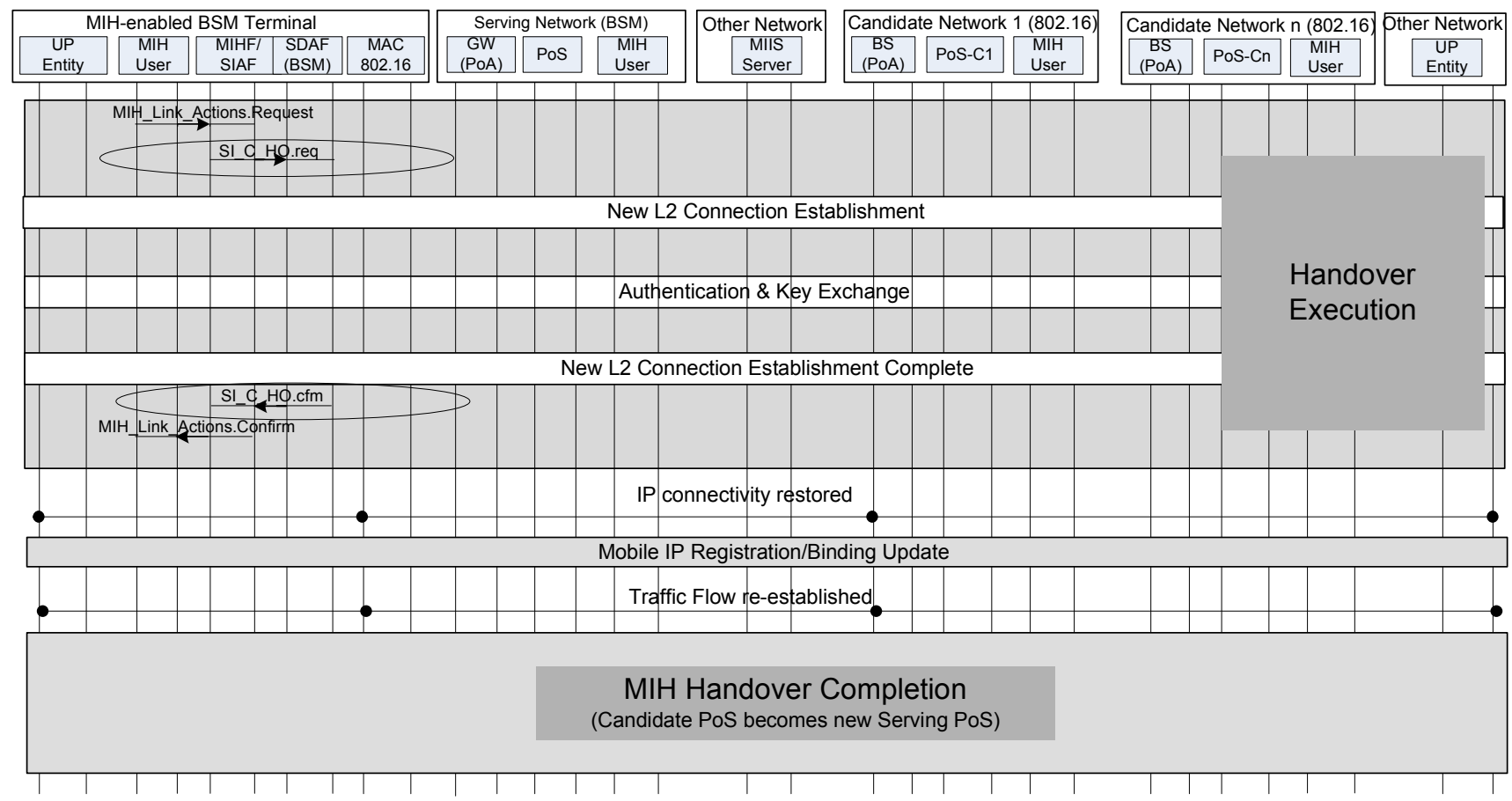

Figure 4bHandover Execution and Completion from MIH-enabled BSM network to 802.16 network

\section{Bibliography}

[1] ETSI TR 101984 V1.2.1 (2007-12), Technical Report, Satellite Earth Stations and Systems (SES); Broadband Satellite Multimedia (BSM); Services and architectures.

[2] ETSI TS 102357 V1.1.1 (2005-05), Technical Specification, Satellite Earth Stations and Systems (SES); Broadband Satellite Multimedia (BSM) Common air interface specification; Satellite Independent Service Access Point (SI-SAP).

[3] Y.F.Hu and P.M.L.Chan, "Mobility Management for BSM", International Workshop on Satellite and Space Communications, IWSSC 2008 .
[4] IEEE Std802.21 Part 21, "Media Independent Handover Services", 2008 .

[5] IEEE Std802.21, '21-06-0620-01-0000-New_section_5_5', May 2006.

[6] IEEE Std802.21, “Clarification of MIH_NMS_SAP”, Nov. 2006.

\section{Acknowledgement}

This work is funded by the EU FP6 Satellite Network of Excellence (SatNExII). 